\title{
OPTIMAL SPAN LENGTH DETERMINATION IN TRANSMISSION SYSTEMS WITH HYBRID AMPLIFICATION
}

\author{
J.D. Ania-Castañón ', I.O. Nasieva ', S.K. Turitsyn ', C. Borsier ${ }^{2}$, and E. Pincemin ${ }^{2}$, \\ ${ }^{1}$ Aston University, School of Engineering and Applied Science, Birmingham B4 7ET, United \\ Kingdom,e-mail: aniacajd@aston.ac.uk \\ ${ }^{2}$ France Telecom R\&D, 2 Aven. Pierre Main, 22307 Lannion, France, e-mail: \\ erwan.pincemin@francetelecom.com
}

Abstract: The existence of an optimal span length for $40 \mathrm{Gbit/s}$ WDM transmission systems with hybrid Raman/EDFA amplification is demonstrated. Optimal lengths are obtained for specific amplifier configurations and different fibre arrangements based on SSMF/DCF and SLA/IDF implementation, using a simple nonlinearity management theory.

\section{INTRODUCTION}

The recent availability of reliable high power laser pumps has made possible the comeback of distributed Raman amplification (DRA) in DWDM transmission systems [1-3]. Compared to traditional lumped amplifier schemes, the DRA improves significantly the optical signal to noise ratio (OSNR). The OSNR margins which are released by the implementation of DRA can be used for extending the transmission distance and/or decreasing the signal power injected into the fibre span (thus limiting the impact of nonlinearities). Combined with dispersion management and Erbium-doped fibre amplification (EDFA), DRA can be used to better control the signal power evolution inside the amplification spans and, thus, nonlinear effects along the optical line, effectively performing "nonlinearity management" $[4,5]$. In this case, the properties of the fibre used (Raman gain, attenuation, effective area, Rayleigh backscattering coefficient) and 
the design of the dispersion map have all to be taken into account when configuring the amplification scheme.

The performance of a given system depends on the trade-off between the requirements of a high OSNR and low nonlinear impairments. Amplifier spacing is a very important design parameter as the amount of noise introduced into the system depends on the span length. Longer amplifier spans are desirable for economical system design. However, in general, they lead to faster OSNR degradation. As we will show in this paper, DRA and a proper nonlinearity management enable system design with a longer optimal amplifier span without degradation of the output OSNR. More specifically, by applying the approach recently developed in $[4,5]$ we investigate here the impact of the periodic span length on the optimal configuration of the amplification scheme in $40 \mathrm{Gbit} / \mathrm{s}$ WDM transmission systems.

\section{THEORY AND SYSTEM CONFIGURATIONS}

Under the assumption that nonlinearities always contribute to the degradation of the performance of the transmission system, nonlinear phase shift (NPS) can be considered as a general measure of the nonlinear impairments [4-8]. The optimal system configuration can then be determined by performing a conditional minimization of the NPS under a fixed OSNR, or vice versa, a maximization of the OSNR under a fixed NPS. In this paper we perform numerical modelling using the average power equations for the Raman pump, signal and noise, in order to find the optimal parameters (gain split between the amplifiers and span length) that allow for the minimization of the NPS under a fixed OSNR. This approach ensures that all important effects, including double Rayleigh backscattering (DRS) noise and pump depletion, are accounted for [5].

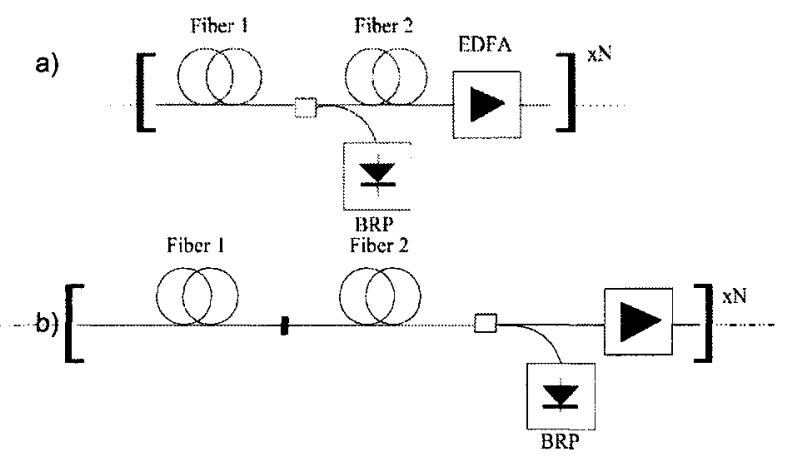

Figure I. System configurations considered 
We focus here on two basic configurations. In both cases, a two-step dispersion map with hybrid Raman/EDFA amplification is considered, but the position of the Raman amplifier is varied. In configuration a), the backward Raman pump is placed immediately after the section of positive dispersion fibre (fibre 1) and an EDFA, with a typical noise figure of $4.5 \mathrm{~dB}$, is used for post-amplification at the end of the span. In configuration b), both amplifiers are placed together at the end of the periodic transmission cell. The combined gain from the two amplifiers compensates exactly for the attenuation of the periodic cell. We consider WDM transmission at $40 \mathrm{Gbit} / \mathrm{s}$ rate with channels equally spaced (by $100 \mathrm{GHz}$ ) and symmetrically distributed at both sides of $1550 \mathrm{~nm}$. Two different dispersion maps are considered for configuration a): one based on standard single-mode fibre (SSMF) with dispersion-compensating fibre (DCF), and another one based on super large area fibre (SLA) with inverse dispersion fibre (IDF). For the configuration b), only the SLA/IDF is considered. A useful parameter to characterize hybrid Raman/EDFA amplification is the ratio $\eta$, defined as the quotient between the on-off gain provided by the DRA and the total gain, both in $\mathrm{dB}$. The length of both positive and negative dispersion fibres within the span are considered as variables, with the length of the negative dispersion fibre always automatically adjusted to compensate exactly for dispersion at the end of each periodic cell. The total transmission distance is fixed to $900 \mathrm{~km}$ over SSMF for the first pair of fibres, or $900 \mathrm{~km}$ total length over SLA+IDF, for the second one. The number of spans varies with the cell length.

\section{RESULTS AND DISCUSSION}

Figure 2 displays NPS in a contour plot versus the length $L$ of the span and the gain ratio $\eta$, for a fixed output OSNR of $22 \mathrm{~dB}$ (the bandwidth for the noise measurement is fixed at $1 \mathrm{~nm}$ ) in configuration a), for both fibre pairs. We can observe that there is a clear optimal length for the periodic span, for which the NPS is minimal, which in this case is about $50 \mathrm{~km}$ for the SSMF/DCF case, and about $90 \mathrm{~km}(60 \mathrm{~km}$ of SLA) for the SLA/IDF scheme. This optimal cell length can be understood as the one that allows us to find the best balance between the two basic variants:

- short cell regime, in which the signal is transmitted in quasi-lossless conditions, so high nonlinearities are produced from even relatively low input powers injected into the spans.

- long cell regime, in which the long distance between amplifiers helps reducing NPS, but leads to an increase of the amplified spontaneous emission (ASE) noise, so the input signal power has to be increased in return, leading to higher nonlinearities. 

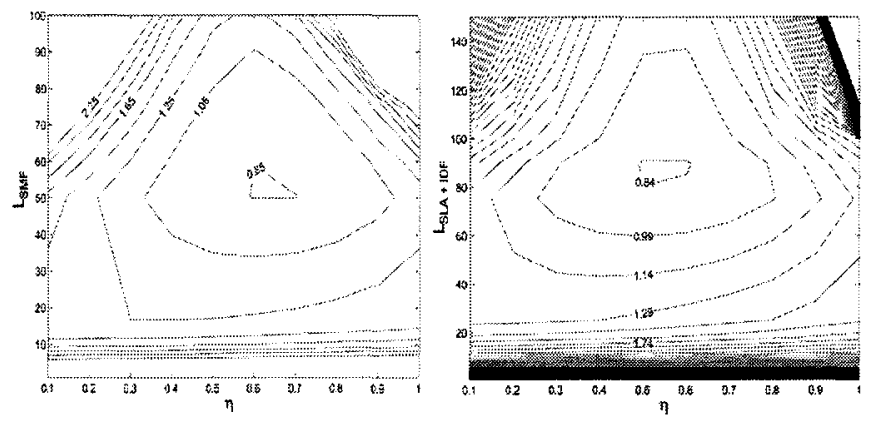

Figure 2. NPS vs. L (in $\mathrm{km}$ ) and $\eta$ for a fixed OSNR of $22 \mathrm{~dB}$ after $900 \mathrm{~km}$ total transmission distance through SSMF/DCF (left) and SLA/IDF (right) with configuration a).

It can also be derived from figure 2 that the optimal $\eta$ varies slightly with the cell length, becoming smaller for long spans. As the span length grows, so does the Raman pump power and gain, and with it the contribution of DRS noise (due to a worse distribution of the gain within the span). In this situation, the gain fraction from the Raman amplifier has to be reduced in order to recover the best possible performance. On the other hand, the relative importance of finding the optimal $\eta$ is increased together with the length of the periodic cell, going from superfluous for the shortest spans, to crucial for the case of $100 \mathrm{~km}$ SSMF section.

It is also important to study how dependent the optimal span length that minimizes the nonlinear impairments is on the targeted output OSNR. By varying the required OSNR, we find that the optimal cell length variation is negligible so it can be considered independent of the output OSNR requirement. On the other hand, the optimal $\eta$ changes slightly, in particular for long span lengths. This phenomenon can be explained by the necessity to avoid the penalties induced by DRS noise for long cells.
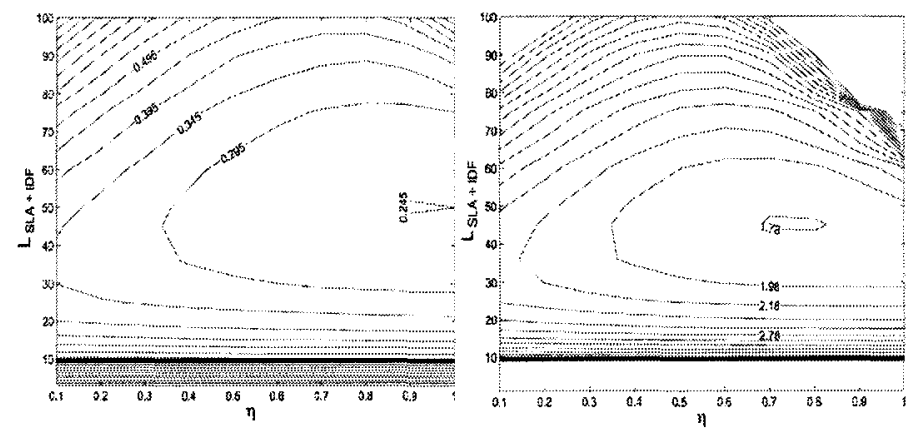

Figure 3. NPS vs. L (in km) and $\eta$ for a fixed OSNR of $22 \mathrm{~dB}$ (left) and $30 \mathrm{~dB}$ (right) after $900 \mathrm{~km}$ total transmission distance SLA/IDF with configuration b). 
Finally, figure 3 shows the results obtained for configuration b): SLA/IDF used with amplifiers located at the end of the transmission cell. The change of configuration has a clear effect on the optimal transmission length, which is reduced to about $50 \mathrm{~km}$ of combined SLA+IDF. For low fixed output OSNR, the optimal amplifier configuration corresponds to values of $\eta$ close to 1 (i.e. full Raman amplification), but when OSNR or span length are increased, so is the Raman gain and with it the effect of DRS, so the optimal configuration requires a higher participation from the EDFA to limit the DRS impact. Finally, we can also observe that, for the same fixed output OSNR, nonlinear impairments are in general lower in scheme b) than in scheme a).

\section{CONCLUSION}

We demonstrate the existence of the optimal span length for $40 \mathrm{Gbit} / \mathrm{s}$ WDM transmission systems considering different fibre mapping arrangements and hybrid Raman/EDFA amplification schemes. The results of the optimization are basically independent of the desired output OSNR, so the optimal span length determined for each system through this method is applicable to a wide range of input signal powers.

\section{REFERENCES}

[1] Christian Rasmussen, Tina Fjelde, Jon Bennike, Fenghai Liu, Supriyo Dey, Benny Mikkelsen, Pavel Mamyshev, Peter Serbe, Paul van der Wagt, Youichi Akasaka, David Harris, Denis Gapontsev, Vladlen Ivshin, and Peter Reeves-Hall, "DWDM 40G transmission over trans-Pacific distance $(10,000 \mathrm{~km})$ using CSRZ-DPSK, enhanced FEC and all-Raman amplified $100 \mathrm{~km}$ UltraWave fiber spans", OFC 2003, PD18, 2003

[2] T. Tsuritani, K. Ishida, A. Agata, K. Shimomura, I. Morita, T. Tokura, H. Taga, T. Mizuochi, and N. Edagawa, "70GHz-spaced $40 \times 42.7 \mathrm{Gbit} / \mathrm{s}$ transmission over $8700 \mathrm{~km}$ using CS-RZ DPSK signal, all-Raman repeaters and symmetrically dispersionmanaged fiber span", OFC 2003, PD23, 2003

[3] M. Mehendale, M. Vasilyev, A. Kobyakov, M. Williams and S. Tsudal, "All-Raman transmission of $80 \times 10 \mathrm{Gbit} / \mathrm{s}$ WDM signals with $50 \mathrm{GHz}$ spacing over $4160 \mathrm{~km}$ of dispersion-managed fibre" Electron. Lett., 38, p. 648, 2002

[4] S.K. Turitsyn, M.P. Fedoruk, V.K. Mezentsev and E.G. Turitsyna, "Theory of optimal power budget in quasi-linear dispersion-managed fibre links", Electron. Lett., 39 (2003), p. 29

[5] J. D. Ania-Castañón, I. O. Nasieva, N. Kurukitkoson, S. K. Turitsyn, C. Borsier and E. Pincemin, "Nonlinearity management in fiber transmission systems with hybrid amplification", Opt. Comm., 233, p. 353, 2004

[6] J.-C. Antona, S. Bigo and J-.P. Faure, "Nonlinear cunulated phase as a criterion to assess performance of terrestrial WDM systems", OFC 2002, WX5, p. 365, 2002 
[7] V. E. Perlin and H. G. Winful, "On trade-off between noise and nonlinearity in WDM systems with Raman amplification”, Proc. OFC 2002, WB1, p. 178, 2002

[8] A. Kobyakov, M. Vasilyev, S. Tsuda, G. Giudice and S. Ten, "Raman noise figure in dispersion-managed fibers", Proc. ECOC 2002, P1.13, 2002 